\title{
Impacts of Revised Quantum Electrodynamics on Fundamental Physics
}

\author{
Bo Lehnert \\ Alfvén Laboratory, Royal Institute of Technology, Stockholm, Sweden \\ Email: bo.lehnert@ee.kth.se
}

How to cite this paper: Lehnert, B. (2018) Impacts of Revised Quantum Electrodynamics on Fundamental Physics. Journal of Electromagnetic Analysis and Applications, 10, 106-118.

https://doi.org/10.4236/jemaa.2018.105008

Received: April 6, 2018

Accepted: May 28, 2018

Published: May 31, 2018

Copyright $\odot 2018$ by author and Scientific Research Publishing Inc. This work is licensed under the Creative Commons Attribution International License (CC BY 4.0).

http://creativecommons.org/licenses/by/4.0/

\section{cc) (i) Open Access}

\begin{abstract}
A revised quantum electrodynamic theory by the author is reconsidered, in respect to the basic concepts established by M. Planck and H.B.G. Casimir on the Zero Point Energy (ZPE) and by A. Einstein on Special Relativity. Attention is given to the new properties of its field equations and their applications. These equations include results not being available from conventional theory and the Standard Model. This concerns the internal structure of elementary particles, such as the photon, the electron, muon and tauon, the $\mathrm{Z}$ boson, and the so called Higgs particle detected in the experiments at the laboratory of CERN. A possible unification of electrodynamics and the strong nuclear force is further provided by the theory. Finally, there are aspects on the expanding universe represented by a new interpretation of dark matter and dark energy in terms of the ZPE.
\end{abstract}

\section{Keywords}

Quantum Electrodynamics, Standard Model and Beyond, Zero Point Energy, Higgs Particle, Unification of Electrodynamics and the Strong Force, Expanding Universe, Dark Matter and Dark Energy

\section{Introduction}

Conventional theoretical physics and its Standard Model have often been successful in its applications, and have sometimes manifested itself in an extremely good agreement with experiments. Examples of this are given in elementary particle physics such as by Ryder [1], as well as in other applications based on Maxwell's equations and quantum mechanics.

Nevertheless there exist areas within which these joint theories do not provide fully adequate descriptions of physical reality. As already stated by Feynman [2], and still having to be taken into account, there are unsolved problems leading to 
difficulties with Maxwell's equations that are not removed by and not directly associated with quantum mechanics. It has at the same time to be remembered that these equations in the vacuum state of empty space have served as guideline and basis for the development of quantum electrodynamics. The latter will therefore become subject to the type of shortcomings of electrodynamics which occur in its conventional form.

It is clear that applications of Maxwell's conventional equations to media which include particles of given electric charge and mass become fully relevant. But an entirely different situation arises when looking into the interior of the same particles, thereby asking for the mechanisms which generate their charges and rest masses. Thus, if the particle interior would be represented by a vacuum state of empty space within which only spinless electromagnetic waves are propagating, how can then something such as charge and rest mass come out of nothing?

An answer to this question is attempted in the revised quantum electrodynamic theory (RQED) by the author [3] [4], being described and elucidated in this context. The latter is especially devoted to the main features, potentialities and impacts of the theory in general terms. Reference is made to earlier reports for the detailed deductions.

\section{Shortcomings of Conventional Theory}

A treatment based on the sourceless vacuum state of empty space, with a vanishing divergence of the electric field strength and basic equations being symmetric in the electric and magnetic field strengths $E$ and $B$, is encumbered with several shortcomings and restrictions.

First, for a photon model this leads to a vanishing spin and a velocity of propagation being exactly equal to the velocity constant $c$ of light, as shown in Ch. 9 of Ref. [3]. Since the photon spin is experimentally confirmed, its incorporation into the field theory would then have to be satisfied either by violating Special Relativity or by making an ad hoc addition of it.

Second, the conventional description of the photon in terms of plane waves cannot account for the needle-like behaviour of the individual photon, as described in Ch. 4.1 and 11 of Ref. [3].

Third, in a steady state a sourceless empty vacuum model further leads to vanishing field strengths. Then there would not exist any rest masses of the particle models, in contradiction to experiments. This is also confirmed by Quigg [5] who states that the symmetry of the field equations of the Standard Model makes them strongly restricted and does not permit masses for leptons and quarks.

A fourth question concerns the Higgs particle concept. One of the first to emphasize the difficulty with the vanishing rest masses in conventional theory was Higgs [6], who proposed a nonlinear mechanism of spontaneous symmetry breaking to overcome this problem. But the theory by Higgs is still confronted 
with some remaining difficulties:

- The theory is based on the spontaneous formation of a heavy boson, but it does not lead to the exact value of its mass, such as that of $125 \mathrm{GeV}$ reported for the particle discovered by CERN [7] [8].

- There are researchers such as M. Turner and S. Glashov who have thrown some doubts upon such a rather complex process, as reported in a review by Mosher [9]. Thus the theory by Higgs consists of a multistage process by which a heavy boson is first being produced, and then decays into all other particles, thereby being given their correct rest masses.

\section{The New Invariant Field Equations}

In a study of the quantum mechanical energy levels of the harmonic oscillator, M. Planck found that there is a nonzero ground level, the Zero Point Energy (ZPE), as being further described in a review by Schiff [10]. The related electromagnetic vacuum fluctuations were investigated by Casimir [11] who predicted that two metal plates will in the vacuum attract each other when being separated by a small spacing. This is due to the fact that only small wavelength: of the fluctuations can exist in the spacing, whereas their full spectrum exerts a force on the outsides of the plates. This tends to push them together. Such a net differential force was first shown to exist by Lamoreaux [12], in using a sensitive torsional pendulum device. Part of these quantum fluctuations also carry electric charge as pointed out by Abbott [13].

Consequently the vacuum is not merely a state of empty space, but includes photon-like fluctuations being each of the frequency $v$ and energy $\frac{1}{2} h v$, and resulting in a real macroscopic pressure. Even if all these fluctuations are at the same low ZPE level, their spatial inhomogeneity can thus produce a pressure gradient. Since the Casimir force is due to a comparatively small differential pressure, the total ZPE can further become much larger, this force only becoming the "top of an isberg".

With these basic concepts as starting points, a revised quantum electrodynamic theory (RQED) can be elaborated as follows. It is first noticed that the Lorentz invariant field equations in a sourceless state are given by d'Alembert where

$$
\square A_{\mu}=\left(\frac{1}{c^{2}} \frac{\partial^{2}}{\partial t^{2}}-\nabla^{2}\right) A_{\mu}=0
$$

in a four-dimensional form with $\mu=1,2,3,4$, standing for the operator in the bracket of Equation (1), and

$$
A_{\mu}=(A, i \phi / c) .
$$

Here $A$ is the magnetic vector potential in three-space, $\phi$ the electrostatic potential, and

$$
c^{2}=1 / \mu_{0} \varepsilon_{0}
$$

where $c$ denotes the velocity constant of light, determined by the magnetic 
permeability $\mu_{0}$ and the dielectric constant $\varepsilon_{0}$.

Taking the electromagnetic sources in the vacuum state into account in a formally correct Lorentz invariant formulation, the field equations have to be modified by adding the four-current density

$$
J_{\mu}=(\boldsymbol{j}, i c \bar{\rho})
$$

to the right-hand member of Equation (1). Here $j$ is the current density and $\bar{\rho}$ the electric charge density in three-space. Consequently

$$
\square A_{\mu}=\mu_{0} J_{\mu} .
$$

The resulting Equation (5) is identical with its corresponding conventional form for a medium containing particles of given electronic charge and mass. But in the present situation it applies instead to a nonzero charge density

$$
\bar{\rho}=\varepsilon_{0} \operatorname{div} \boldsymbol{E}
$$

and a nonzero energy density [3]

$$
w_{s}=\frac{1}{2} \bar{\rho}(\phi+C \cdot A)
$$

in the vacuum state; where $C$ is a later defined vector.

Since $J_{\mu}$ is relativistically invariant in transitions between inertial frames, the form (4) has to satisfy the condition

$$
\boldsymbol{j}^{2}-c^{2} \bar{\rho}^{2}=\text { const. }
$$

The constant in this equation has further to vanish because $J_{\mu}$ disappears when there are no sources of Equations (6) and (7). This leads to the final form

$$
J_{\mu}=\bar{\rho}(\boldsymbol{C}, i c)
$$

where

$$
\boldsymbol{C}^{2}=c^{2}
$$

and $C$ becomes a velocity vector with a modulus equal to $c$. Equation (10) thus defines the modulus of the vector $\boldsymbol{C}$ which can have several components, such as in the case of cylindrical waves.

In a three-dimensional representation the new extended Lorentz invariant field equations of the vacuum state are thus given by

$$
\begin{gathered}
c^{2} \operatorname{curl} \boldsymbol{B}=(\operatorname{div} \boldsymbol{E}) \boldsymbol{C}+\frac{\partial \boldsymbol{E}}{\partial t} \\
\operatorname{curl} \boldsymbol{E}=-\frac{\partial \boldsymbol{B}}{\partial t}
\end{gathered}
$$

where

$$
\begin{gathered}
\boldsymbol{B}=\operatorname{curl} \boldsymbol{A}, \quad \operatorname{div} \boldsymbol{B}=0 \\
\boldsymbol{E}=\phi+\frac{\partial \boldsymbol{A}}{\partial t}, \quad \operatorname{div} \boldsymbol{E}=\bar{\rho} / \varepsilon_{0} .
\end{gathered}
$$

These equations are also gauge invariant, because their new added parts include the field strength $E$ as in conventional theory, i.e. in terms of a gauge 
transformation of the potentials $\boldsymbol{A}$ and $\phi$.

The corresponding equations of conventional theory describing the sourceless vacuum state of empty space mentioned in Section 2 are obtained from the present set (11)-(14) in the special and strongly restricted case where $\operatorname{div} \boldsymbol{E}=0$.

In analogy with conventional theory as described by Heitler [14], the present quantized field equations become identical with the original ones, in which the potentials and current densities are replaced by their expectation values. Relevant and specific quantum conditions, such as that of the spin, can then be imposed on the corresponding general solutions.

Equations (5)-(14) have new features and lead to new results, not being available from conventional theory:

- The nonzero electric field divergence does not only generate net particle charges, but also intrinsic charges of both signs within the volume of an elementary particle. Hereby $\operatorname{div} \boldsymbol{E}$ acts somewhat like a hidden variable.

- The theory thus includes sources in the vacuum state, resulting in broken symmetry of the field strengths $\boldsymbol{E}$ and $\boldsymbol{B}$.

- In conventional theory, the Lorentz invariance is generally connected with transitions between the frames of plane electromagnetic waves. In the present theory the Lorentz invariance is extended into a multidimensional form represented by the velocity vector $C$. Thus the velocity constant $c$ of Equation (3) does not become identical with the velocity of propagation of an individual photon.

- The general solution in steady-state particle-shaped geometry can be obtained from a generating function. It produces the related and self-consistent forms of the corresponding potentials $(\boldsymbol{A}, \phi)$ and the charge density $\bar{\rho}$. Radially convergent generating functions result in particle models with vanishing net electric charge, whereas divergent such functions lead to a nonzero net charge, a point-charge-like geometry, and a modified renormalization process.

- The source terms of the present theory also lead to a volume force density

$$
\boldsymbol{f}=\bar{\rho}(\boldsymbol{E}+\boldsymbol{C} \times \boldsymbol{B}) .
$$

Generally its total field

$$
\boldsymbol{E}_{t}=\boldsymbol{E}+\boldsymbol{C} \times \boldsymbol{B}
$$

becomes nonzero, whereas part of the electric contribution $E$ is out balanced by the magnetic contribution $\boldsymbol{C} \times \boldsymbol{B}$.

\section{New Results on Elementary Particles}

The applications of RQED theory on elementary particles concern characteristic dimensions of $10^{-16} \mathrm{~m}$ and less. All particle models have source terms due to broken symmetry which leads to steady states with nonzero rest masses, and often with an associated spin. There are both particles with net electric charge such as the electron, muon and tauon, and those with vanishing net charge such as the photon, $\mathrm{Z}$ boson, the so called Higgs particle, and the neutrino. The net 
charge arises from the integral of $\bar{\rho}$ in Equation (6) over the particle volume, and the rest mass from the integral of $w_{s} / c^{2}$ of Equation (7). A new important feature is here the intrinsic electric charges $+Q_{i}$ and $-Q_{i}$, which arise from integrals of $\bar{\rho}$ over limited parts of the particle volumes.

The present particle models are axisymmetric, with the exception of the neutrino for which chirality has to be taken into account.

\subsection{The Photon}

The resulting model of the individual photon obtains the following features:

- It is first observed that the Standard Model leads to a vanishing integrated spin, as shown in Ref. [15] [16] and in Ch. 9 of Ref. [3], thus being in contradiction with experiments. Such a spinless photon of plane-wave geometry would propagate exactly at the velocity $c$ of Equation (3).

- In fact, the plane-wave concept becomes infinitely extended over space and is in itself an unphysical representation of the individual photon. An acceptable form in a limited region of space is on the other hand provided by a wave-packet solution in cylindrical geometry.

- The present theory results in a screw-shaped configuration of cylindrical geometry which includes a spin at the expense of a very small reduction of its phase and group velocities from the velocity constant $c$. This reduction occurs only in the ninth decimal as compared to the value of $c$, see Ref. [17] [18] [19] and Ch. 11 of Ref. [3]. The nonzero spin is then associated with a very small but nonzero rest mass.

- This model contributes to the understanding of the observed needle radiation of individual photons, as well as of the wave-particle dualism, the photoelectric effect, and of two-slit experiments [17] [18] [19] [20] [21].

- In a light beam consisting of propagating photons with unidirectional spins, the equivalent angular momentum of its core is replaced by a real angular momentum in its boundary layer. This is confirmed by experiments as described in Ch. 12.2.4 of Ref. [3].

\subsection{The Electron, Tauon and Muon}

Particles with net integrated charge, such as the electron, muon and tauon, are represented by models as follows:

- These models are obtained from divergent generating functions in pointcharge-like geometry, as shown in Ref. [22] and Ch. 7 of Ref. [3].

- The divergence problem of infinite self-energy in such a geometry can be tackled by a modified process of renormalization. A conventional way is described by Ryder [1] among others, implying that the divergence is outbalanced by adding extra ad hoc counter terms to the Lagrangian, thereby obtaining a finite result from a finite difference between two "infinities". In this theory, an alternative way is chosen in which the "infinity" of the system is outbalanced by the "zero" of a counterfactor, to give a final convergent 
result [3] [22] [23] [24].

- The deductions then result in a net charge $q_{0}=Q_{0} f_{p}$, where $Q_{0}=\left(\varepsilon_{0} c h\right)^{1 / 2}=8.86 \times 10^{-19} \mathrm{C}$ and $f_{p}$ is a dimensionless profile factor of the particle geometry, and $Q_{0}$ is related to the fine-structure constant. This gives an example where relativity is linked with quantum mechanics through the present theory.

- A variational analysis applied on the factor $f_{p}$ finally results in a minimum charge $q_{0} \cong 1.602 \times 10^{-19} \mathrm{C}$, within an uncertainty of only a few percent as compared to the experimental value of $e$ [3] [14] [25].

- The Lorentz invariance of the electron radius can be formally satisfied in the present theory, also in cases where this radius is allowed to shrink to that of a point-charge-like state [3].

- It is expected that the charged particles are prevented from "exploding" by their electrostatic eigenforce, due to the counteracting magnetostatic force [3] [25].

The models of these leptons also include rest masses:

- The source terms due to broken symmetry result for steady states into nonzero rest masses and an associated spin.

- According to the present theory, the product of the rest mass $m_{0}$ and the magnetic moment $M_{0}$ of these particles becomes

$$
m_{0} M_{0}=(c h / 4 \pi)\left[1+\left(e^{2} / 4 \pi \varepsilon_{0} c h\right)\right]=8.455 \times 10^{-54} \mathrm{~kg} \cdot \mathrm{A} \cdot \mathrm{m}^{2}
$$

with good agreement compared to experimental data, as shown in Ref. [26] and in Ch. 7.74 of Ref. [3].

- This theory also presents solutions with the same geometry but opposite signs of the integrated field quantities, thus applying to antimatter such as the positron as shown in Ch. 6.3.3 in Ref. [3].

\subsection{The Z Boson and the CERN Particle}

There are also particles with vanishing net charge, the models of which can be obtained in terms of a convergent generating function:

- One example is the $\mathrm{Z}$ boson for which a rest mass of $91 \mathrm{GeV}$ becomes connected with a characteristic radius of about $10^{-18} \mathrm{~m} \mathrm{[26]} \mathrm{[27]} \mathrm{[28]} \mathrm{[29]}$ [30].

- A superposition of two Z boson configurations with opposite magnetostatic potentials leads to a composite purely electrostatic and unstable system. The latter has no net charge, no eigenmagnetic field, no spin, and a minimum mass of $125 \mathrm{GeV}$ within limits of a few percent [27] [28] [29] [30]. This deduced particle model has all the basic properties of the particle detected at CERN [7] [8]. But it has no relation to the theory by Higgs.

- When feeding an increasing energy into this system, such a minimum value would become the first to be reached in the case of a heavy particle mass. 


\subsection{Possible Unification of Electrodynamics and the Strong Force}

So far the internal particle structure has been considered in this context. We now turn to the mutual short-range interaction between particles, in particular under the influence of their intrinsic charge geometry. As an example, we consider a tentative model of the neutron, given by three individual quarks which are mutually bound to each other to form a triangular configuration [31], as described in current investigations [1] [32]. In a first simplified treatment, equal models are used for each of the quarks.

The following points are to be observed concerning the model of an individual quark:

- A convergent and separable generating function is given by the Ansatz

$$
G(\rho, \theta)=R(\rho) \cdot T(\theta), \quad R=\rho^{\gamma} \mathrm{e}^{-\rho}, \quad T=(\sin \theta)^{\alpha}
$$

in a frame $(r, \theta, \varphi)$ of spherical coordinates with $\rho=r / r_{0}, r_{0}$ as a characteristic radial dimension, and all relevant quantities being independent of $\varphi$. The disposable parameters $\gamma>0$ and $\alpha>0$ represent various but similar profile shapes. The general form of the field quantities which result from $G$ for various values of $\gamma$ and $\alpha$, as well as for minor convergent changes of the form (18), are expected to be similar as far as orders of magnitude are concerned. When studying a detailed outcome of the theory, we shall therefore restrict ourselves here to the special case $\gamma=\alpha=1$ as a first demonstration.

- In the present triangular configuration, each quark is assumed to have a mass $m=5.57 \times 10^{-28} \mathrm{~kg}$ being a third of the neutron mass. Here $m$ becomes equal to $w_{s} / c^{2}$ of Equation (7) being integrated over the quark volume.

- Integration of the local charge density $\bar{\rho}$ of Equation (6) over various parts of the quark volume further results in large positive and negative intrinsic charges $\pm Q_{i}$ where $Q_{i} / e=19.9$. Thus $Q_{i}$ is an order of magnitude larger than the elementary charge $e$.

- Since $\boldsymbol{C} \times \boldsymbol{B}$ outbalances part of $\boldsymbol{E}$ in Equations (15) and (16), the total field strength $\boldsymbol{E}_{t}$, only becomes noticeable in the innermost region of the quark volume, i.e. within a core which only occupies about 3 percent of this volume. The remaining 97 percent are thus in a force-free state. With the Ansatz (18) the integrated force with density (15) therefore becomes comparatively small. This is in particular the case when comparing such an eigenforce to the force that arises between two mutually interacting quarks in the present triangular configuration.

- It is expected that a small modification of the generating function (18) can eliminate the remaining small integrated eigenforce contribution within the core. This can be done by adding a small part being radially convergent and having the correct sign. The local force density (15) then still becomes nonzero, but its integral over the quark volume would vanish to result in a force-free state of the eigenforce.

Turning further to the mutual interaction between two quarks the following points have to be observed: 
- The mutual interaction is governed by the intrinsic charges and their radial distributions within the quark volume. The result $\left(Q_{i} / e\right)^{2}=396$ implies that the force due to the field $E$ becomes more than two orders of magnitude larger than that arising between two elementary charges. But the real situation is more complex, due to the added influence of the magnetostatic part $\boldsymbol{C} \times \boldsymbol{B}$ which partly outbalances that of $\boldsymbol{E}$.

- The total interaction between two quarks is thus of a short-range character, and becomes about two orders of magnitude larger than that arising between two elementary charges at the same particle distance.

- The mutual quark force, and the corresponding work of it, are determined in terms of a variable distance between the quark centra. This is done by considering the force due to the local intrinsic charge density $\bar{\rho}$ of quark (I) in combination with the radially reversed field $\boldsymbol{E}_{t}$ of quark (II).

- When gradually shrinking the distance of the quark centra, a corresponding potential distribution is being traversed. This is obtained in the form of an outer potential barrier, followed by an intermediate well, and ending at an additional barrier. The mutual interaction between the quarks thus becomes stable and attractive.

- An application to the neutron then leads to a well depth of about 7.7 MeV. This is rather close to the binding energy of $8 \mathrm{MeV}$ obtained from experiments reported by Bethe [33]. Since the additional net charges of the proton are of the magnitude of the elementary charge, a similar result is expected to hold also for the proton.

- For the triangular quark model of the neutron and proton, an outer radius of about $3 \times 10^{-15} \mathrm{~m}$ and a core radius of about $10^{-15} \mathrm{~m}$ are obtained. These values are compatible with a nuclear radius of $1.5 \times 10^{-15} \mathrm{~m}$ given by experiments [33].

- An uncertainty is due to the geometry of the intrinsic charge distribution which can become perturbed by the mutual quark forces.

- The examples given in this context are thus connected with some uncertainties concerning the obtained numerical results and their accuracy. Nevertheless the general outcome could still hold, as far as orders of magnitude are concerned. If therefore gives a clear indication that it can become possible to unify the fundamental phenomena of electrodynamics and the strong nuclear force.

\section{New Aspects on the Expanding Universe}

The basis formed by the Zero Point Energy (ZPE) and the Casimir force also have an impact on the physics of the expanding universe. This is particularly the case at the dimensions of the observable universe, having a radius of about $10^{26} \mathrm{~m}$.

\subsection{The Frequency Spectrum of the Zero Point Energy}

Several investigators such as Riess and Turner [34] and Heitler [14] have thrown doubts upon the conventional theory on the vacuum energy and its related 
frequency distribution. The following points indicate that some fundamental parts are lacking [35] [36]:

- The conventional theory on the frequency spectrum is unacceptable, because it is underdetermined, treats all states with the same statistical probability of unity, and leads to an infinite total energy density.

- This problem is eliminated by including a Boltzmann factor into a revised special form which is deduced in terms of conventional statistical theory, being applied on the ZPE fluctuations.

\subsection{A New Interpretation of Dark Matter and Dark Energy}

On the basis of ZPE, dark matter and dark energy have been interpreted in a new way [35] [36]:

- Dark matter then originates from the ZPE density and leads to a contracting gravitational force acting on a ZPE photon cloud.

- Dark energy originates on the other hand from the ZPE density gradient which leads to an expansive force.

- Dark matter and dark energy are thus due to a common spectrum and spatial distribution. This can resolve the related coincidence problem of nearly equal magnitudes of dark matter and energy, as well as the difficulty with the cosmological constant.

- An application is made here to the observable universe as a whole, with presently estimated proportions of dark matter, dark energy, and normal matter as given by astronomers and other researchers such as Linde [37], Riess and Turner [34] and Linder and Perlmutter [38]. An equilibrium state of the ZPE photon gas then becomes reconcilable with the radial dimension $10^{26} \mathrm{~m}$ of the observable universe. This can be taken as a support of the present approach.

- This approach can become a largest-scale complement to the effects of black holes on the scale of galaxies.

- The Casimir force detected in earlier experiments with a small but nonzero spacing between the metal plates is comparatively small, as being the result of a minor differential effect. The total ZPE photon pressure can therefore turn out to be much larger. To complete the experimental determinations of the ZPE density, it is proposed to make measurements on the maximum Casimir force which arises between two plates of different metals at a vanishing air gap [39]. Then there only remains an equivalent air gap represented by the small electromagnetic skin depth of the photons. The latter can be varied by choosing plates of different metals.

\section{Summary of Main Results}

With the basic concepts established by M. Planck, H.B.G. Casimir and A. Einstein as starting points, a revised quantum electrodynamic theory has been elaborated by the author. It leads to new results in fundamental physics, not 
being available from conventional theory and the Standard Model:

- There is a non-zero rest mass of the leptons and a number of other elementary particles which both have zero and nonzero net electric charges.

- The individual photon is represented by a screw-shaped cylindrical geometry in which there is a nonzero spin being associated with a very small but nonzero rest mass.

- The cylindrical geometry of this photon model, which has limited dimensions in the directions being perpendicular to the direction of propagation, provides the individual photon with the property of needle radiation.

- The electron, muon and tauon have deduced minimum net electronic charges. These are equal to the experimentally given elementary charge $e$, within an uncertainty of a few percent.

- The deduced minimum of a composite boson mass becomes equal to the measured value of $125 \mathrm{GeV}$ for the particle detected by CERN, within an uncertainty of a few percent.

- There are large intrinsic electrical charges in the interior of the particle volumes, having both polarities. These charges lead to a mutual short-range interaction between quarks, being of the same magnitude as that of the strong nuclear force.

- This quark interaction can become attractive and stable, thereby being associated with a potential well depth that becomes nearly equal to the binding energy of $8 \mathrm{MeV}$ for the neutron and proton. This provides a clear indication for a unification of the electrodynamic force with the strong nuclear force.

- The density of the Zero Point Energy photons has related effects which can explain the behaviour of dark matter and dark energy and become consistent with given cosmological data of the expanding universe.

\section{Acknowledgements}

The author is indebted to Professors Kai Siegbahn, Hans Wilhelmsson, Jan Scheffel, Nils Abramson, Einar Tennfors and Dmitri Rabounski for many valuable discussions on the manifolds of problems related to this review. My sincere thanks also to M.Sc. Kerstin Holmström for skillful and indefatigable assistance with a manifold of manuscripts of the present theory.

\section{References}

[1] Ryder, L.H. (1996) Quantum Field Theory. 2nd Edition, Cambridge University Press, Cambridge. https://doi.org/10.1017/CBO9780511813900

[2] Feynman, R.P. (1964) Lectures in Physics: Mainly Electromagnetism and Matter, Mathematics in Science and Engineering. Addison-Wesley, Reading, MA, p. 28-1.

[3] Lehnert, B. (2014) Revised Quantum Electrodynamics. In: Dvoeglazov, V.V., Ed., Contemporary Fundamental Physics, Nova Science Publishers, New York, 1-154.

[4] Lehnert, B. (2014) Some Consequences of Zero Point Energy. Journal of Electromagnetic Analysis and Applications, 6, 319-327. 
https://doi.org/10.4236/jemaa.2014.610032

[5] Quigg, C. (2008) The Coming Revolution in Particle Physics. Scientific American, 298, 46-53. https://doi.org/10.1038/scientificamerican0208-46

[6] Higgs, P.W. (1966) Spontaneous Symmetry Breakdown without Massless Bosons. Physical Review, 145, 1156-1168. https://doi.org/10.1103/PhysRev.145.1156

[7] Aad, G., et al. (2012) ATLAS Collaboration. Observation of a New Particle in the Search for the Standard Model Higgs Boson with the ATLAS Detector at the LHC. Physics Letters B, 716, 1-29. https://doi.org/10.1016/j.physletb.2012.08.020

[8] Chatrchyan, S., et al. (2012) CMS Collaboration. Observation of a New Boson at a Mass of $125 \mathrm{GeV}$ with the CMS Experiment at the LHS. Physics Letters B, 716, 30-61. https://doi.org/10.1016/j.physletb.2012.08.021

[9] Mosher, D. (2007) Greatest Mysteries: What Causes Gravity? http://www.livescience.com/1770-greatest-mysteries-gravity.html

[10] Schiff, L.I. (1949) Quantum Mechanics. McGraw-Hill Book Comp., Inc., New York-Toronto-London, Ch. IV, Sec. 13.

[11] Casimir, H.B.G. (1948) On the Attraction between Two Perfectly Conducting Plates. Proceedings of the Koninklijke Nederlandse Akademie van Wetenschappen, 51, 793-795.

[12] Lamoreaux, S.K. (1997) Demonstration of the Casimir Force in the 0.6 to $6 \mu \mathrm{m}$ Range. Physical Review Letters, 78, 5-8. https://doi.org/10.1103/PhysRevLett.78.5

[13] Abbott, L. (1988) The Mystery of the Cosmogical Constant. Scientific American, 258, 106-113. https://doi.org/10.1038/scientificamerican0588-106

[14] Heitler, W. (1954) The Quantum Theory of Radiation. Clarendon Press, Oxford, Appendix, p. 409.

[15] Lehnert, B. (2008) Failure of Maxwell's Equations as a Basis for a Photon Model in the Vacuum State. International Review of Physics (I.R.E.PHY), 2, 337-340.

[16] Lehnert, B. (2013) On the Angular Momentum and the Rest Mass of the Photon. Journal of Plasma Physics, 79, 1133-1135. https://doi.org/10.1017/S002237781300069X

[17] Lehnert, B. (2005) Screw-Shaped Light in Extended Electromagnetics. Physica Scripta, 72, 359-365. https://doi.org/10.1238/Physica.Regular.072a00359

[18] Lehnert, B. (2006) Photon Physics in Revised Electromagnetics. Progress in Physics, 2, 78-85.

[19] Lehnert, B. (2007) Joint Wave-Particle Properties of the Individual Photon. Progress in Physics, 4, 104-108.

[20] Lehnert, B. (2011) The Individual Photon in Two-Slit Experiments. International Review of Physics, 5, 15-18.

[21] Lehnert, B. (2006) Boundary Conditions and Spin of a Dense Light Beam. Physica Scripta, 74, 139-144. https://doi.org/10.1088/0031-8949/74/1/020

[22] Lehnert, B. (2010) Deduced Fundamental Properties of the Electron. International Review of Physics, 4, 1-6.

[23] Lehnert, B. (2008) A Revised Electromagnetic Theory with Fundamental Applications. The National Library of Sweden, Stockholm, Bogoljubov Institute for Theoretical Physics, Kiev 2008.

[24] Lehnert, B. and Scheffel, J. (2002) On the Minimum Elementary Charge of an Extended Electromagnetic Theory. Physica Scripta, 65, 200-207. https://doi.org/10.1238/Physica.Regular.065a00200 
[25] Lehnert, B. and Höök, J. (2010) An Electron Model with Elementary Charge. Journal of Plasma Physics, 76, 419-428.

[26] Lehnert, B. (2016) Revised Quantum Electrodynamics in a Condensed Form. Journal of Modern Physics, 7, 1316-1319. https://doi.org/10.4236/jmp.2016.711117

[27] Lehnert, B. (2013) Higgs-Like Particle Due to Revised Quantum Electrodynamics. Progress in Physics, 3, 31-32.

[28] Lehnert, B. (2014) Mass-Radius Relations of $\mathrm{Z}$ and Higgs-Like Bosons. Progress in Physics, 10, 5-7.

[29] Lehnert, B. (2015) Some Elucidations of the Theory on Revised Quantum Electrodynamics. Journal of Modern Physics, 6, 1695-1700. https://doi.org/10.4236/jmp.2015.611171

[30] Lehnert, B. (2015) Minimum Mass of a Composite Boson. Journal of Modern Physics, 6, 2074-2079. https://doi.org/10.4236/jmp.2015.614214

[31] Lehnert, B. (2017) Intrinsic Particle Charges and the Strong Force. Journal of Modern Physics, 8, 1053-1066. https://doi.org/10.4236/jmp.2017.87067

[32] Riordan, M. and Zajk, W.A. (2006) The First Few Seconds. Scientific American, 294, 34-41. https://doi.org/10.1038/scientificamerican0506-34A

[33] Bethe, H.A. (1947) Elementary Nuclear Theory. John Wiley and Sons, Inc., New York, and Chapman and Hall, Ltd., London, Ch. 2, 118.

[34] Riess, A.G. and Turner, M.S. (2004) From Slowdown to Speedup. Scientific American, 290, 50-55. https://doi.org/10.1038/scientificamerican0204-62

[35] Lehnert, B. (2009) On Dark Energy and Matter of the Expanding Universe. Progress in Physics, 2, 77-82.

[36] Lehnert, B. (2013) Dark Energy and Dark Matter as due to Zero Point Energy. Journal of Plasma Physics, 79, 327-334. https://doi.org/10.1017/S0022377812001055

[37] Linde, A. (1994) The Self-Reproducing Inflationary Universe. Scientific American, 32, 32-39. https://doi.org/10.1038/scientificamerican1194-48

[38] Linder, E. and Perlmutter, S. (2007) Dark Energy: The Decade Ahead. Physics World, 20, 24-30. https://doi.org/10.1088/2058-7058/20/12/29

[39] Lehnert, B. (2014) Extended Analysis of the Casimir Force. Progress in Physics, 10, 76-78. 